\title{
Relative Effects of Different Non-vitamin K antagonist Oral AntiCoagulants on Global Thrombotic Status in Atrial Fibrillation
}

Mohamed Farag, MBBCh, MSc 1,2 Maria Niespialowska-Steuden, MRCP 1,3, Osita Okafor, BSc, MBBS ${ }^{1}$, Benjamin Artman, BSc, MBBS ${ }^{1}$, Manivannan Srinivasan, MBBS, MD ${ }^{1}$, Arif Khan, MBBS ${ }^{1}$, Keith Sullivan, $\mathrm{PhD}^{2}$, David Wellsted, PhD ${ }^{2}$, Diana A Gorog MBBS, MD, PHD, FRCP 1,2,3,

1. Department of Cardiology, East and North Hertfordshire NHS Trust, Hertfordshire, UK

2. Postgraduate Medical School, University of Hertfordshire, UK

3. National Heart \& Lung Institute, Imperial College, London, UK

Short title: Effects of NOACs on Global Thrombotic Status in AF Funding: East and North Hertfordshire NHS Trust

Conflict of interest: D.A.G. is related through family to a company director in Thromboquest $L t d$, but has no financial involvement or equity interest in, and has received no financial assistance, support, or grant from the aforementioned company. The rest of authors have nothing to declare.

\section{Correspondence to:}

Prof. Diana A Gorog

Imperial College, London, UK

Tel +44 (0)2070348934

Fax $+44(0) 1707247688$

d.gorog@imperial.ac.uk 


\section{Abstract}

Non-vitamin K antagonist oral anticoagulants (NOACs) reduce the risk of thromboembolism in patients with atrial fibrillation (AF). There has been no head-to-head comparison of the effect of these agents on ex-vivo thrombotic and thrombolytic status. Enhanced platelet reactivity and impaired endogenous thrombolysis are risk factors for recurrent thrombotic events. We aimed to assess the comparative effect of NOACs and warfarin using an ex-vivo test of thrombosis and thrombolysis. Eighty patients with newly diagnosed non-valvular AF were tested before, and after being established on apixaban $(n=20)$, dabigatran $(n=20)$, rivaroxaban $(n=20)$, or warfarin $(n=20)$. Thrombotic status was assessed with the automated, point-of-care Global Thrombosis Test (GTT), that assesses both platelet reactivity and endogenous thrombolysis from native blood. The time taken to form an occlusive thrombus (occlusion time, OT), and the time required to restore flow through endogenous thrombolysis (lysis time, LT) were measured. All anticoagulants caused OT prolongation compared to baseline (apixaban $403 \pm 102$ s vs. $496 \pm 125 s, p=0.006$; dabigatran $471 \pm 106 s$ vs. $656 \pm 165$ s, $p<0.00001$; rivaroxaban $381 \pm 119$ s vs. $579 \pm 158, p<0.00001$; warfarin $420 \pm 145$ s vs. $604 \pm 124$ s, p<0.00001). Apixaban reduced LT from baseline (1895[1702-2167]s vs. $1435[347-1990] s ;$ $p=0.006$ ). A trend for LT reduction was seen with other NOACs (dabigatran 1594[1226-2069]s vs. $1539[561-2316] s, p=0.499$; rivaroxaban $2085[1366-2428]$ s vs. $1885[724-2420] s, p=0.295$ ) but not warfarin (1490[1206-1960]s vs. 1776[1545-2334], $p=0.601)$. Our results suggest that NOACs and warfarin have a similar favourable effect on reducing platelet reactivity. All NOACs exhibited a trend towards enhancing endogenous thrombolytic status, although this was significant only for apixaban. This raises the possibility of using NOACs to enhance impaired endogenous fibrinolysis in patients at high thrombotic risk. 
Key words: atrial fibrillation, anticoagulant drugs, thromboembolism, fibrinolysis, platelet function tests

Words: 256

\section{List of abbreviations}

$A F=$ atrial fibrillation 
GTT = Global Thrombosis Test

$\mathrm{LT}=$ lysis time

NVAF $=$ non-valvular atrial fibrillation

NOACs $=$ non-vitamin $\mathrm{K}$ antagonist oral anticoagulants

OT = occlusion time

PFTs $=$ platelet function tests

VKAs $=$ vitamin $\mathrm{K}$ antagonists 


\section{Background}

Non-vitamin $\mathrm{K}$ antagonist oral anticoagulants (NOACs) are licensed for the thromboprophylaxis of stroke and systemic embolism in patients with non-valvular atrial fibrillation (NVAF) and are non-inferior to warfarin [1-4] ${ }^{1-4}$. There has been no head-to-head comparison of the effect of these agents on ex vivo thrombotic and thrombolytic status. Enhanced platelet reactivity and impaired endogenous thrombolysis are risk factors for recurrent thrombotic events $[5,6]{ }^{5,6}$. The rate of major bleeding associated with NOACs compared to warfarin is variable. Apixaban demonstrated a significantly lower rate of major bleeding compared to warfarin [7] ${ }^{7}$, whereas dabigatran at the $150 \mathrm{mg}$ BID dose and rivaroxaban were similar to warfarin in $\operatorname{RELY}[4]^{4}$ and Rocket $A F[8]^{8}$, although subsequent registries suggest that major bleeding is infrequent with rivaroxaban $[9,10]^{9,10}$. NOACs reduce the occurrence of intracranial bleeding significantly compared to warfarin. However, there has been concern that dabigatran, particularly at high dose, is associated with more gastrointestinal bleeding $[11,12]^{11,12}$.

All NOACs exert their anticoagulant effects through direct or indirect thrombin inhibition. Data suggest that NOACs do not affect platelet reactivity to common agonists (ADP, arachidonic acid, collagen) [13-15] ${ }^{13-15}$, and their effect on platelets may be confined to inhibiting $\alpha$-thrombin-induced platelet aggregation. Even the latter is controversial, as dabigatran, but not rivaroxaban, enhanced thrombin receptor activating peptide (TRAP)induced platelet aggregation as measured by multiple electrode aggregometry [16] ${ }^{16}$. However, conventional point-of-care platelet function tests (PFTs) cannot be used to monitor NOACs, since these tests measure platelet reactivity to various soluble platelet agonists and neither NOACs nor warfarin have an effect on this $[13,14]{ }^{13,14}$. Further, as these tests use 
citrate-anticoagulated blood, thrombin is not generated from activated platelets and the important procoagulant activity of platelets is not measured [17] ${ }^{17}$. Most available in vitro PFTs using citrate-anticoagulated blood do not assess the contribution of thrombin generated by activated platelets, despite the recognition that thrombin is the key mediator of venous thrombus formation [18 ${ }^{18}$. It was thus essential to compare the effect of NOACs using noncitrated blood.

Enhanced platelet reactivity and pro-coagulant state are not the sole determinants of lasting arterial occlusion in patients with ischemic stroke. Spontaneous endogenous thrombolysis is an important defence mechanism against tissue damage from lasting vessel occlusion [5,19$21]^{5,19-21}$. In patients with acute stroke, spontaneous arterial recanalization was observed in $17-24 \%$ of patients [22-24] ${ }^{22-24}$, and was strongly related to improved functional outcomes [23] ${ }^{23}$. In 185 patients with stroke, ex vivo endogenous thrombolysis was significantly impaired, compared to normal volunteers [25] ${ }^{25}$. A recent review article highlighted the importance of endogenous fibrinolysis in determining clinical outcome, concluding that global assays, assessing proaggregatory and fibrinolytic pathways, could aid in identifying impaired fibrinolysis as a potential target for pharmacological modulation [5] ${ }^{5}$. The biological assessment of thrombotic risk should therefore not only include assessment of platelet function, but also endogenous thrombolytic status. The Global Thrombosis Test (GTT) (Thromboquest Ltd., London, UK), assesses platelet reactivity, coagulation (thrombin generation) and spontaneous endogenous thrombolysis from a native (non-anticoagulated) blood sample. In this test, thrombin generation from shear-activated platelets and fibrinstabilization of the initial platelet aggregates play a major role in determining the measured occlusion time. Therefore, it was highly suited to assess the relative and absolute effects of 
NOACs and warfarin on overall thrombotic status. Our aim was to assess the comparative effect of NOACs and warfarin using an ex-vivo test of thrombosis and thrombolysis. 


\section{Methods}

\section{Study design and population}

The study was approved by the local research ethics committee. Eighty patients with newly diagnosed, haemodynamically stable NVAF were enrolled in a single-centre, prospective observational study after obtaining written informed consent, each taking one of 4 anticoagulant regimens. Patients were either naive to oral anticoagulation or had been previously anticoagulated with warfarin or NOAC, but had temporarily discontinued this (for more than 4 weeks). Exclusion criteria were as follows; less than 18 years of age, active sepsis or infective illness within the last month, known active malignancy, bleeding diathesis, anticoagulation at the time of initial sampling, need for combined antiplatelet and anticoagulant therapy, blood dyscrasia (platelets $<100 \times 10^{9} / \mathrm{L}$, haemoglobin $<80 \mathrm{~g} / \mathrm{L}$, international normalized ratio $>1.4$, activated partial thromboplastin time more than twice the upper limit of normal, leukocyte count $<3.5 \times 10^{9} / \mathrm{L}$, neutrophil count $<1 \times 10^{9} / \mathrm{L}$ ), and alcohol consumption above recommended safe levels (i.e. more than 21 units per week for males, or more than 14 units per week for females) due to the potential effects of high alcohol levels on platelet reactivity. Baseline demographics and clinical characteristics were obtained from the patients and from review of case notes and electronic records. Compliance with NOACs was considered adequate if the pill compliance was $\geq 80 \%$ for at least two consecutive weeks prior to repeat sampling.

\section{Anticoagulant regimens}

The choice of oral anticoagulant was decided by the clinical team looking after the patient. Recruited patients who received vitamin $\mathrm{K}$ antagonist (VKA) therapy, were given warfarin to 
maintain the international normalised ratio (INR) at 2.5 (range 2.0-3.0), based on local standard of care. Patients who received a NOAC, were given one of the following drugs: apixaban, a twice-daily dose of $5 \mathrm{mg}$ orally (or $2.5 \mathrm{mg}$ twice-daily in patients with any two or more of the following characteristics; age $\geq 80$ years, weight $\leq 60 \mathrm{~kg}$, or serum creatinine level $\geq 133 \mu \mathrm{mol} / \mathrm{L}$ ); dabigatran, a twice-daily dose of $150 \mathrm{mg}$ orally (or $110 \mathrm{mg}$ twice-daily in patients aged $\geq 80$ years or at high risk of bleeding complications); rivaroxaban, a once-daily dose of $20 \mathrm{mg}$ orally (or $15 \mathrm{mg}$ once daily in patients with creatinine clearance of 30-49 $\mathrm{mL} / \mathrm{min}$ ), based on local standard of care. All patients, but in particular those receiving rivaroxaban were advised of the importance of taking their medication with their main meal to achieve high bioavailability [26] ${ }^{26}$. Patients were advised to avoid aspirin and any other over the counter medications and those at risk of ulcerative gastrointestinal disease or bleeding could receive prophylactic treatment with proton-pump inhibitors.

\section{Blood sampling}

Blood samples were taken from an antecubital vein on 2 occasions: 1) baseline, and before initiating oral anticoagulation, and 2) after being established on stable anticoagulation for at least two weeks, and in the case of warfarin, once the INR was therapeutic for at least a week. Samples were taken approximately 3 hours after NOACs or warfarin. Fasting was not required. All samples were taken from an antecubital vein using an 18-G butterfly cannula, avoiding prolonged tourniquet time and using a 2-syringe technique, whereby the first $5 \mathrm{ml}$ was used for standard blood tests (biochemistry, full blood count) and the second sample used for GTT analysis. The patient was positioned in close proximity to the GTT such that the blood sample was introduced into the GTT within 15 seconds of withdrawal and the automated measurement begun. 


\section{Assessment of thrombotic status}

Assessment of thrombotic status was performed using the Global Thrombosis Test (GTT, Thromboquest Ltd., London, UK), an automated point-of-care test that assesses both platelet reactivity and endogenous thrombolysis from a native, non-anticoagulated blood sample. The technique assesses the time taken to form an occlusive platelet thrombus (occlusion time, OT), and in the second phase of the test, measures the time required to dissolve the thrombus formed in the first phase, through endogenous thrombolysis, which thereby restores flow (lysis time, LT). The principle of the GTT has previously been described in detail $[5,27,28]^{5,27,28}$. In brief, blood is allowed to flow in a conical plastic tube through narrow gaps where fibrinstabilized platelet aggregates to occlude the gaps, and the instrument measures the time (d) between 2 consecutive blood drops by an optical sensor in the space downstream. This time interval increases gradually as flow slows down and at an arbitrary point ( $d \geq 15 \mathrm{~s}$, before reaching complete occlusion), the first end-point of the measurement is displayed (occlusion time [OT], in seconds). Restart of blood flow after occlusion is due to spontaneous thrombolysis, and the second end-point of the measurement is displayed (lysis time [LT], in seconds). The pre-set cut-off time for OT was $900 \mathrm{sec}$ and for LT was $6000 \mathrm{sec}$. The intra-assay and inter-assay coefficients of variation (CV) were assessed by repeated sampling on 4 subjects.

\section{Statistical analysis}

This is a pilot, exploratory study and patients acted as self controls, such that bias or confounders were minimised. Samples of 20 patients per group were felt to be sufficient to give representative results when evaluating self-controls. $[29,30]^{29,30}$. 
Data are presented as mean and standard deviation (when data normally distributed) or median and inter-quartile range [IQR] (non-normally distributed). Where necessary, log transformations were employed. Dichotomous variables were compared using chi-square test or Fisher's exact test, as appropriate. The Kruskal-Wallis one-way analysis of variance was used to assess the differences in OT and LT between the groups and in response to different anticoagulants. Analyses were performed with Stata version 11.2 (StataCorp, College Station, TX, USA), blinded to drug allocation. 


\section{Results}

The characteristics of our study population are shown in Table 1. In our hands the intra-assay CV was $\mathrm{OT}=10 \%$ and for $\mathrm{LT}=6 \%$ and the inter-assay $\mathrm{CV}$ was $\mathrm{OT}=8 \%$ and for $\mathrm{LT}=9 \%$. Distribution of OT, prior to anticoagulation, is shown in Figure 1. There was no significant difference in baseline OT between groups assigned to different oral anticoagulants (OACs) (Table 1), and any subtle difference was likely to be due to subtle differences in clinical characteristics between the groups as well as the play of chance. Compared to baseline, OT was significantly prolonged (showing reduced platelet reactivity) by all OACs (apixaban $403 \pm 102$ s vs. $496 \pm 125$ s, $p=0.006$; dabigatran $471 \pm 106$ s vs. $656 \pm 165 s, p<0.00001$; rivaroxaban $381 \pm 119$ s vs. $579 \pm 158$, $p<0.00001$; warfarin $420 \pm 145$ s vs. $604 \pm 124 s, p<0.00001$ ) [Figure 2]. Apixaban caused relatively less prolongation of OT from baseline than the other NOACs or warfarin (change in OT with apixaban $94 \pm 135$ s, dabigatran $185 \pm 149$ s, rivaroxaban $199 \pm 104$ s, warfarin $184 \pm 149$ s; $p=0.062)$.

Distribution of LT, prior to anticoagulation, is shown in Figure 1. There was no difference in baseline LT between groups (Table 1). Compared to baseline, LT was reduced (showing enhanced endogenous thrombolysis) after oral anticoagulation compared to baseline LT [Figure $1 \mathrm{C}$ and $\mathrm{D}$, and Figure 3]. Whilst the median values of LT before and after OACs are numerically similar, the different sum ranks result in a significant $p$ value (1755[1386-2314]s vs. $1753[807-2246]$ s; $p=0.026$, sum ranks 1987 and 1093). The LT graphs (Figure 1 C and D) demonstrate a significant shift in distribution. This was due predominantly to apixaban, which significantly reduced LT from baseline (1895[1702-2167]s vs. 1435[347-1990]s; $p=0.006$ ) compared to a non-significant difference in LT observed with other NOACs or warfarin (dabigatran 1594[1226-2069]s vs. 1539[561-2316]s, $p=0.499$; rivaroxaban 2085[1366-2428]s 
vs. $1885[724-2420] s, p=0.295$; warfarin $1490[1206-1960]$ s vs. $1776[1545-2334], p=0.601$ ) [Figure 3].

All the variables in Table 1 were interrogated for effects on baseline OT or LT using parametric and non-parametric tests, as appropriate, and showed no significant relationship. Patients in the dabigatran group were younger than in other groups, and patients in the rivaroxaban group had higher $\mathrm{CHA}_{2} \mathrm{DS}_{2}$-VASc score than in other groups, otherwise groups were well matched. 


\section{Discussion}

All the oral anticoagulants studied reduced platelet reactivity, although apixaban caused relatively little effect. All NOACs, but not warfarin, exhibited a trend towards enhancing endogenous thrombolytic status, although this was significant only for apixaban. This raises the possibility of using NOACs to enhance impaired endogenous fibrinolysis in patients at high thrombotic risk.

The main limitation of our study is predominantly the small sample size, and therefore the potential impact of small differences between groups confounding the results. Patients in the dabigatran group were younger than in other groups. In addition, the GTT is designed to measure global thrombotic status of blood subjected to high flow state (i.e. arterial thrombosis), so it may not provide a reliable assessment of low flow prothrombotic state, such as that which occurs in the left atrium of patients with AF. Although only apixaban caused significant reduction in LT, a trend in LT reduction was observed with the other NOACs and perhaps if the sample size had been sufficiently large, an effect on LT with the other oral anticoagulants may have been observed. Oral food intake is known to affect the absorption of rivaroxaban [26] ${ }^{26}$, but since this was not monitored, we cannot be sure that maximal peak levels of the active metabolite had been reached at the time of sampling. Finally, we did not monitor the pharmacokinetics of the NOACs to ensure maximum bioavailability at the time of sampling, and variation in this may have influenced the results. The pharmacodynamics of NOACs are relatively similar, in contrast to their pharmacokinetics, which are largely specific to each individual agent [31] ${ }^{31}$. As the GTT does not use any chemical reagents (such as platelet agonists) and the physical characteristics of the individual cartridges (and hence flow and shear rates) are identical, reliability and reproducibility of the test depends entirely on 
how the blood sample is obtained. Untrained phlebotomy, prolonged venous stasis, or deviation from the protocol described by the manufacturer could result in variable test results. Our blood samples were obtained by clinicians well-versed in the technique, who took care to avoid prolonged tourniquet time. The patient was seated next to the GTT instrument so that blood could be immediately introduced into the instrument. Whilst this may be regarded by some as a limitation of the GTT, we feel it also makes the assessment far more physiological. The GTT is particularly well-adapted to investigate the role of thrombin inhibitors such as NOACs, for many reasons, primarily because the OT in the GTT can assess the contribution of thrombin [32] ${ }^{32}$. Most conventional platelet function tests which require blood to be collected into citrate-anticoagulant, reduce calcium levels such that there is inhibition of thrombin generation from activated platelets and the important procoagulant activity of platelets is not measured. This is not the case with the GTT, where, thrombin generation from shear-activated platelets and fibrin-stabilization of the initial platelet aggregates play a major role in determining the measured occlusion time. Secondly, the GTT is, to our knowledge, the only available technique to allow assessment of fibrinolysis of a platelet-rich thrombus, under high shear conditions, that reflect those in an atherosclerotic arterial milieu, which was the aim of our study. However, the GTT cannot currently be recommended for influencing clinical decision making, as further evidence is needed from studies showing that decision making based on GTT results can favourably influence outcomes.

Unlike VKAs, NOACs have been designed to inhibit specific single targets in the coagulation cascade. Apixaban and rivaroxaban are selective inhibitors of factor $\mathrm{X}$ (factor $\mathrm{Xa}$ ) and do not require a cofactor (such as anti-thrombin) for their activity. Dabigatran is a direct competitive inhibitor of thrombin, the final effector in blood coagulation. These agents have no direct 
effect on platelet aggregation, but indirectly inhibit platelet aggregation induced by thrombin. Our study shows that all oral anticoagulants prolong OT (showing reduction in platelet reactivity). Apixaban was associated with relatively less prolongation of OT than other oral anticoagulants and importantly, was the only anticoagulant regimen that significantly reduced LT (showing enhanced endogenous thrombolysis). Importantly, we did not observe a clear difference in effect between thrombin (dabigatran) or factor Xa (rivaroxaban and apixaban) inhibitors, since the effects of dabigatran and rivaroxaban were similar on OT and LT. We would have expected the effects of rivaroxaban and apixaban to be similar. The bioavailability of rivaroxaban is increased if taken with food, unlike the case with apixaban which is relatively independent of food-intake [26] ${ }^{26}$. Possible insufficient food intake with rivaroxaban may have led to a reduced observed effect of this drug on thrombotic parameters, compared to apixaban. It is also possible, that there is no difference between NOACs, and observed differences with apixaban compared to other NOACs is simply due to chance, or due to differences in baseline characteristics between groups. These could include the relatively high frequency of diabetics and the slightly lower BMI in the apixaban group, the latter may have subtly influenced pharmacokinetics and pharmacodynamics, although this is unlikely given that no grossly obese individuals were included. Finally, compliance was assessed by pill-count, but since we did not measure drug concentrations, compliance and absorption cannot be confirmed and may have contributed to differing results between groups. The relatively small increase in OT with apixaban compared to other oral anticoagulants may indeed explain the observed lower incidence of major bleeding in large trials with this agent $[7,33-35]^{7,33-35}$. 
The effect of medications on endogenous fibrinolysis has hitherto been unimpressive. Prior work has shown that aspirin at low doses does not alter LT, whereas more potent antiplatelet agents such as clopidogrel have a modest effect on LT [36] ${ }^{36}$. Previously, in a study of patients with coronary disease, treatment with the PAR-1 inhibitor vorapaxar, in addition to standard care with antiplatelet therapy, favourably reduced LT [37] ${ }^{37}$. Our current work lends further support to the notion that agents that inhibit thrombin, but not antiplatelet agents, can exert a favourable effect on endogenous thrombolysis. The observed favourable effect of apixaban on endogenous thrombolysis compared to other NOACs in our study is not fully understood. This finding however supports the antithrombotic properties of apixaban and can help to better understand the mechanism(s) of action of this drug.

There has been a call for "personalised" antithrombotic treatment, to tailor medications to the individual, to optimise outcomes and reduce unwanted complications $\left[38,39{ }^{38,39}\right.$. Large clinical trials are warranted to assess whether assessment of thrombotic status is useful, in addition to clinical risk scores, to not only predict stroke risk, but also assess response to oral anticoagulant therapy. Whether patients with prolonged LT derive greater advantage from NOACs compared to VKAs, or whether they derive potentially greater benefit from a particular NOAC, such as apixaban, remains to be seen. Our early, provocative data calls for further large studies to assess the clinical usefulness of measuring the response to oral anticoagulants, and how this correlates with observed effects of OACs on ex vivo tests of thrombosis. 


\section{References}

1. Ruff CT, Giugliano RP, Braunwald E, Hoffman EB, Deenadayalu N, Ezekowitz MD, Camm AJ, Weitz JI, Lewis BS, Parkhomenko A and others. Comparison of the efficacy and safety of new oral anticoagulants with warfarin in patients with atrial fibrillation: a meta-analysis of randomised trials. Lancet 2014;383(9921):955-62.

2. Bruins Slot KM, Berge E. Factor Xa inhibitors versus vitamin $\mathrm{K}$ antagonists for preventing cerebral or systemic embolism in patients with atrial fibrillation. Cochrane Database Syst Rev 2013;8:CD008980.

3. Miller CS, Grandi SM, Shimony A, Filion KB, Eisenberg MJ. Meta-analysis of efficacy and safety of new oral anticoagulants (dabigatran, rivaroxaban, apixaban) versus warfarin in patients with atrial fibrillation. Am J Cardiol 2012;110(3):453-60.

4. Connolly SJ, Ezekowitz MD, Yusuf S, Eikelboom J, Oldgren J, Parekh A, Pogue J, Reilly PA, Themeles E, Varrone J and others. Dabigatran versus warfarin in patients with atrial fibrillation. N Engl J Med 2009;361(12):1139-51.

5. Okafor O, Gorog D. Endogenous fibrinolysis: an important mediator of thrombus formation and cardiovascular risk. J Am Coll Cardiol 2015;65:1683-1699.

6. Parodi G, Marcucci R, Valenti R, Gori AM, Migliorini A, Giusti B, Buonamici P, Gensini GF, Abbate $R$, Antoniucci $D$. High residual platelet reactivity after clopidogrel loading and longterm cardiovascular events among patients with acute coronary syndromes undergoing $\mathrm{PCl}$. JAMA 2011;306(11):1215-23.

7. Granger CB, Alexander JH, McMurray JJ, Lopes RD, Hylek EM, Hanna M, Al-Khalidi HR, Ansell $\mathrm{J}$, Atar D, Avezum $\mathrm{A}$ and others. Apixaban versus warfarin in patients with atrial fibrillation. $\mathrm{N}$ Engl J Med 2011;365(11):981-92.

8. Patel MR, Mahaffey KW, Garg J, Pan G, Singer DE, Hacke W, Breithardt G, Halperin JL, Hankey GJ, Piccini JP and others. Rivaroxaban versus warfarin in nonvalvular atrial fibrillation. N Engl J Med 2011;365(10):883-91.

9. Camm AJ, Amarenco P, Haas S, Hess S, Kirchhof P, Kuhls S, van Eickels M, Turpie AG, Investigators X. XANTUS: a real-world, prospective, observational study of patients treated with rivaroxaban for stroke prevention in atrial fibrillation. Eur Heart J 2015.

10. Eerenberg ES, Middeldorp S, Levi M, Lensing AW, Büller HR. Clinical impact and course of major bleeding with rivaroxaban and vitamin $\mathrm{K}$ antagonists. J Thromb Haemost 2015;13(9):1590-6.

11. Hernandez I, Baik SH, Piñera A, Zhang Y. Risk of bleeding with dabigatran in atrial fibrillation. JAMA Intern Med 2015;175(1):18-24.

12. Graham DJ, Reichman ME, Wernecke M, Zhang R, Southworth MR, Levenson M, Sheu TC, Mott $\mathrm{K}$, Goulding MR, Houstoun M and others. Cardiovascular, bleeding, and mortality risks in elderly Medicare patients treated with dabigatran or warfarin for nonvalvular atrial fibrillation. Circulation 2015;131(2):157-64.

13. Eller T, Busse J, Dittrich M, Flieder T, Alban S, Knabbe C, Birschmann I. Dabigatran, rivaroxaban, apixaban, argatroban and fondaparinux and their effects on coagulation POC and platelet function tests. Clin Chem Lab Med 2014;52(6):835-44.

14. Zemer-Wassercug N, Haim M, Leshem-Lev D, Orvin KL, Vaduganathan M, Gutstein A, Kadmon E, Mager A, Kornowski R, Lev EL. The effect of dabigatran and rivaroxaban on platelet reactivity and inflammatory markers. J Thromb Thrombolysis 2015.

15. Martischnig AM, Mehilli J, Pollak J, Petzold T, Fiedler AK, Mayer K, Schulz-Schüpke S, Sibbing D, Massberg S, Kastrati A and others. Impact of Dabigatran versus Phenprocoumon on ADP Induced Platelet Aggregation in Patients with Atrial Fibrillation with or without Concomitant Clopidogrel Therapy (the Dabi-ADP-1 and Dabi-ADP-2 Trials). Biomed Res Int 2015;2015:798486. 
16. Olivier CB, Weik P, Meyer M, Weber S, Anto-Michel N, Diehl P, Zhou Q, Geisen U, Bode C, Moser M. TRAP-induced platelet aggregation is enhanced in cardiovascular patients receiving dabigatran. Thromb Res 2015.

17. Gorog DA, Jeong YH. Platelet function tests: why they fail to guide personalized antithrombotic medication. J Am Heart Assoc 2015;4(5).

18. Gorog DA, Fuster V. Platelet function tests in clinical cardiology: unfulfilled expectations. J Am Coll Cardiol 2013;61(21):2115-29.

19. Ose KJ, Blebea J, Fowl RJ, Kempczinski RF. Spontaneous thrombolysis of native artery occlusions. Ann Vasc Surg 1993;7(5):463-9.

20. Onohara T, Takahashi I, Nishizaki T, Wakasugi K, Matsusaka T, Kume K. Superior mesenteric vein thrombosis due to diverticulitis and spontaneous thrombolysis after ilio-cecal resection. A case report. J Cardiovasc Surg (Torino) 2000;41(2):307-9.

21. Kim HS, Huh CW, Kim DS, Mok JH, Kim IS, Kim SH. Spontaneous Thrombolysis of Multiple Thrombi at Distal Region of Hypoplastic Vertebral Artery After Stent-assisted Angioplasty on Vertebral Artery Origin Stenosis: Angiographic Follow-up. J Cerebrovasc Endovasc Neurosurg 2014;16(3):281-6.

22. Kassem-Moussa H, Graffagnino C. Nonocclusion and spontaneous recanalization rates in acute ischemic stroke: a review of cerebral angiography studies. Arch Neurol 2002;59(12):1870-3.

23. Rha JH, Saver JL. The impact of recanalization on ischemic stroke outcome: a meta-analysis. Stroke 2007;38(3):967-73.

24. Alexandrov AV, Bladin CF, Norris JW. Intracranial blood flow velocities in acute ischemic stroke. Stroke 1994;25(7):1378-83.

25. Taomoto K, Ohnishi $H$, Kuga $Y$, Nakashima K, Ichioka T, Kodama $Y$, Kubota H, Tominaga T, Hirose $T$, Hayashi $M$ and others. Platelet function and spontaneous thrombolytic activity of patients with cerebral infarction assessed by the global thrombosis test. Pathophysiol Haemost Thromb 2010;37(1):43-8.

26. Stampfuss J, Kubitza D, Becka M, Mueck W. The effect of food on the absorption and pharmacokinetics of rivaroxaban. Int J Clin Pharmacol Ther 2013;51(7):549-61.

27. Saraf S, Christopoulos C, Salha IB, Stott DJ, Gorog DA. Impaired endogenous thrombolysis in acute coronary syndrome patients predicts cardiovascular death and nonfatal myocardial infarction. J Am Coll Cardiol 2010;55(19):2107-15.

28. Sharma S, Farrington K, Kozarski R, Christopoulos C, Niespialowska-Steuden M, Moffat D, Gorog DA. Impaired thrombolysis: a novel cardiovascular risk factor in end-stage renal disease. Eur Heart J 2013;34(5):354-63.

29. Julious SA. Sample size of 12 per group rule of thumb for a pilot study. Pharmaceutical Statistics 2005;4(4):287-291.

30. Hertzog MA. Considerations in determining sample size for pilot studies. Res Nurs Health 2008;31(2):180-91.

31. Mueck W, Schwers S, Stampfuss J. Rivaroxaban and other novel oral anticoagulants: pharmacokinetics in healthy subjects, specific patient populations and relevance of coagulation monitoring. Thromb J 2013;11(1):10.

32. Yamamoto J, Inoue N, Otsui K, Ishii H, Gorog DA. Global Thrombosis Test (GTT) can detect major determinants of haemostasis including platelet reactivity, endogenous fibrinolytic and thrombin generating potential. Thromb Res 2014;133(5):919-26.

33. Hylek EM, Held C, Alexander JH, Lopes RD, De Caterina R, Wojdyla DM, Huber K, Jansky P, Steg $P G$, Hanna $M$ and others. Major bleeding in patients with atrial fibrillation receiving apixaban or warfarin: The ARISTOTLE Trial (Apixaban for Reduction in Stroke and Other Thromboembolic Events in Atrial Fibrillation): Predictors, Characteristics, and Clinical Outcomes. J Am Coll Cardiol 2014;63(20):2141-7. 
34. Connolly SJ, Eikelboom J, Joyner C, Diener HC, Hart R, Golitsyn S, Flaker G, Avezum A, Hohnloser SH, Diaz R and others. Apixaban in patients with atrial fibrillation. N Engl J Med 2011;364(9):806-17.

35. Lip GY, Larsen TB, Skjøth F, Rasmussen LH. Indirect comparisons of new oral anticoagulant drugs for efficacy and safety when used for stroke prevention in atrial fibrillation. J Am Coll Cardiol 2012;60(8):738-46.

36. Saraf $S$, Wellsted D, Sharma S, Gorog DA. Shear-induced global thrombosis test of native blood: pivotal role of ADP allows monitoring of P2Y12 antagonist therapy. Thromb Res 2009;124(4):447-51.

37. Rosser G, Tricoci P, Morrow D, Christopoulos C, Niespialowska-Steuden MN, Kozarski R, Wilcox R, Gorog DA. PAR-1 antagonist vorapaxar favorably improves global thrombotic status in patients with coronary disease. J Thromb Thrombolysis 2014;38(4):423-9.

38. Delgado MG, Corte JR, Sáiz A, Calleja S. Recurrent lacunar ischaemic stroke due to resistance to antiplatelet treatment: examining the need for personalised antithrombotic therapy. Neurologia 2015;30(6):376-378.

39. Helms TM, Duong G, Zippel-Schultz B, Tilz RR, Kuck KH, Karle CA. Prediction and personalised treatment of atrial fibrillation-stroke prevention: consolidated position paper of CVD professionals. EPMA J 2014;5(1):15.

40. Puurunen MK, Kiviniemi T, Schlitt A, Rubboli A, Dietrich B, Karjalainen P, Nyman K, Niemelä M, Lip GY, Airaksinen KE. CHADS2, CHA2DS2-VASC and HAS-BLED as predictors of outcome in patients with atrial fibrillation undergoing percutaneous coronary intervention. Thromb Res 2014;133(4):560-6. 
Table 1: Baseline Patient Characteristics

\begin{tabular}{|c|c|c|c|c|c|c|}
\hline & $\begin{array}{l}\text { Overall } \\
\text { Group } \\
(n=80)\end{array}$ & $\begin{array}{l}\text { Apixaban } \\
(n=20)\end{array}$ & $\begin{array}{l}\text { Dabigatran } \\
(n=20)\end{array}$ & $\begin{array}{c}\text { Rivaroxaban } \\
(n=20)\end{array}$ & $\begin{array}{l}\text { Warfarin } \\
(n=20)\end{array}$ & $\begin{array}{c}\text { p } \\
\text { Value }\end{array}$ \\
\hline Age, yrs & $72 \pm 12$ & $72 \pm 10$ & $65 \pm 11$ & $78 \pm 9$ & $74 \pm 15$ & 0.006 \\
\hline Male & $49(61)$ & $13(65)$ & $15(75)$ & $10(50)$ & $11(55)$ & 0.375 \\
\hline Body mass index & $29.4 \pm 11.3$ & $27.4 \pm 8.0$ & $30.0 \pm 19.6$ & $29.4 \pm 7.0$ & $29.2 \pm 4.2$ & 0.998 \\
\hline \multicolumn{7}{|l|}{ Atrial Fibrillation } \\
\hline Persistent & $49(61)$ & $12(60)$ & $15(75)$ & $9(45)$ & $13(65)$ & 0.267 \\
\hline Paroxysmal & $31(39)$ & $8(40)$ & $5(25)$ & $11(55)$ & $7(35)$ & 0.267 \\
\hline $\mathrm{CHA}_{2} \mathrm{DS}_{2}$-VASc score & $2.9 \pm 1.7$ & $2.9 \pm 1.8$ & $2.1 \pm 1.6$ & $3.7 \pm 1.7$ & $3.0 \pm 1.5$ & 0.030 \\
\hline HAS-BLED score & $1.6 \pm 1.1$ & $1.3 \pm 0.7$ & $1.6 \pm 0.9$ & $2.3 \pm 1.5$ & $1.5 \pm 1.2$ & 0.352 \\
\hline Smoking & $11(14)$ & $4(20)$ & $5(25)$ & $1(5)$ & $1(5)$ & 0.397 \\
\hline Prior CAD & $20(25)$ & $4(20)$ & $4(20)$ & $6(30)$ & $6(30)$ & 0.468 \\
\hline Diabetes mellitus & $10(13)$ & $5(25)$ & $3(15)$ & $1(5)$ & $1(5)$ & 0.170 \\
\hline Hypertension & $49(61)$ & $12(60)$ & $11(55)$ & $13(65)$ & $13(65)$ & 0.901 \\
\hline
\end{tabular}




\begin{tabular}{|c|c|c|c|c|c|c|}
\hline $\begin{array}{l}\text { Chronic kidney disease } \\
(\text { eGFR<60) }\end{array}$ & $1(1)$ & 0 & 0 & 0 & $1(5)$ & 0.801 \\
\hline \multicolumn{7}{|l|}{ Concomitant medications * } \\
\hline Beta-blocker & $27(34)$ & $9(45)$ & $4(20)$ & $9(45)$ & $5(25)$ & 0.424 \\
\hline Calcium antagonist & $22(28)$ & $6(30)$ & $6(30)$ & $5(25)$ & $5(25)$ & 0.657 \\
\hline ACE inhibitor & $17(21)$ & $8(40)$ & $7(35)$ & $6(30)$ & $4(20)$ & 0.675 \\
\hline Statin & $21(26)$ & $5(25)$ & $6(30)$ & $5(25)$ & $5(25)$ & 0.740 \\
\hline Digoxin & $5(6)$ & $2(10)$ & $1(5)$ & $1(5)$ & $1(5)$ & 0.948 \\
\hline Proton pump inhibitor & $6(7)$ & $2(10)$ & $1(5)$ & $1(5)$ & $2(10)$ & 0.828 \\
\hline \multicolumn{7}{|c|}{ Blood tests on initial sampling } \\
\hline Haemoglobin $(\mathrm{g} / \mathrm{L})$ & $137 \pm 16$ & $138 \pm 16$ & $141 \pm 13$ & $133 \pm 17$ & $134 \pm 18$ & 0.315 \\
\hline Haematocrit (\%) & $41 \pm 5$ & $42 \pm 5$ & $43 \pm 4$ & $39 \pm 5$ & $40 \pm 6$ & 0.099 \\
\hline Platelet count (x 109/L) & $215 \pm 63$ & $220 \pm 62$ & $217 \pm 33$ & $209 \pm 80$ & $214 \pm 71$ & 0.960 \\
\hline Creatinine ( $\mu \mathrm{mol} / \mathrm{L})$ & $87 \pm 28$ & $82 \pm 18$ & $87 \pm 14$ & $92 \pm 24$ & $87 \pm 46$ & 0.738 \\
\hline Fibrinogen $(\mathrm{g} / \mathrm{L})$ & $4.4 \pm 1.6$ & $3.6 \pm 1.0$ & $4.8 \pm 1.9$ & $4.6 \pm 1.6$ & $4.6 \pm 1.4$ & 0.076 \\
\hline Baseline OT (mean \pm SD) & $419 \pm 122 \mathrm{~s}$ & $403 \pm 102 s$ & $471 \pm 106 s$ & $381 \pm 119 s$ & $420 \pm 145 s$ & 0.111 \\
\hline Baseline LT (median/IQR) & $\begin{array}{c}\text { 1755[1386- } \\
2314] \mathrm{s}\end{array}$ & $\begin{array}{c}\text { 1895[1702- } \\
2167] \mathrm{s}\end{array}$ & $\begin{array}{c}\text { 1594[1226- } \\
2069] s\end{array}$ & $\begin{array}{c}\text { 2085[1366- } \\
2428] s\end{array}$ & $\begin{array}{c}\text { 1490[1206- } \\
1960] \mathrm{s}\end{array}$ & 0.103 \\
\hline
\end{tabular}


Values are mean (standard deviation) or $\mathrm{n}(\%)$.

CAD: coronary artery disease; for explanation of $\mathrm{CHA}_{2} \mathrm{DS}_{2}$-VASc risk scoring in AF and HAS-BLED bleeding risk score please see reference [40] ${ }^{40}$; eGFR: estimated glomerular filtration rate (The Modification of Diet in Renal Disease [MDRD] formula).

* No patients were taking concomitant antiplatelet medications.

Normal values: haemoglobin $130-180 \mathrm{~g} / \mathrm{L}$ in adult males and $115-165 \mathrm{~g} / \mathrm{L}$ in adult females; haematocrit $40-52 \%$ in adult males and $36-47 \%$ in adult females; platelet count $150-400 \times 10^{9} / \mathrm{L}$; creatinine $60-$ $110 \mu \mathrm{mol} / \mathrm{L}$ in adult males and 45-90 $\mu \mathrm{mol} / \mathrm{L}$ in adult females; fibrinogen $2-4 \mathrm{~g} / \mathrm{L}$. 
Figure 1: Distribution of OT and LT before and after oral anticoagulants

(A) Baseline OT, and before initiating oral anticoagulation, (B) OT after being established on stable oral anticoagulants, (C) Baseline LT, and before initiating oral anticoagulation, and (D) LT after being established on oral anticoagulant. In NVAF patients receiving oral anticoagulants, OT was significantly prolonged (showing reduced platelet reactivity) compared to baseline OT, as evidenced by a rightward shift (higher OT, representing reduced platelet reactivity). In NVAF patients, LT was significantly reduced (showing enhanced endogenous thrombolysis) after oral anticoagulants compared to baseline LT, as evidenced by a leftward shift (lower LT, representing faster lysis).

(A)

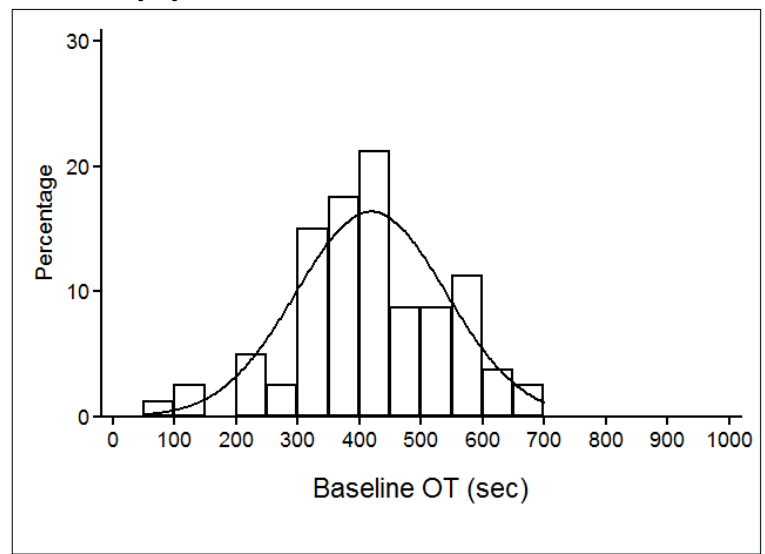

(C)

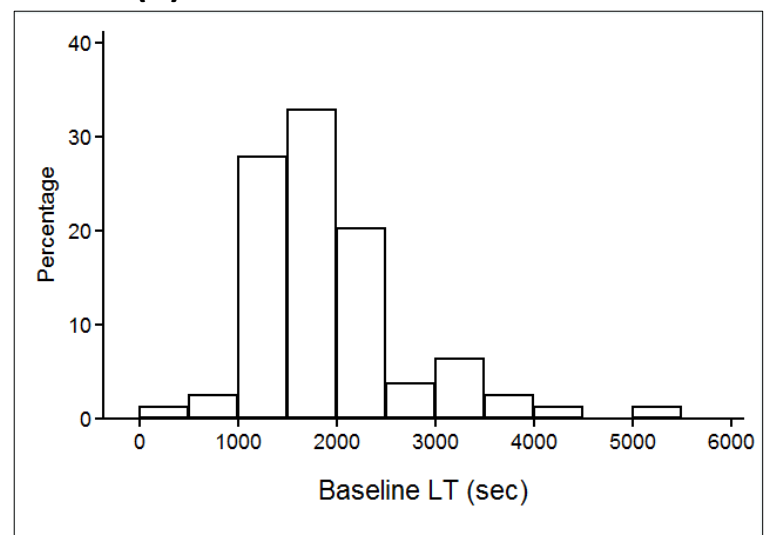

(B)

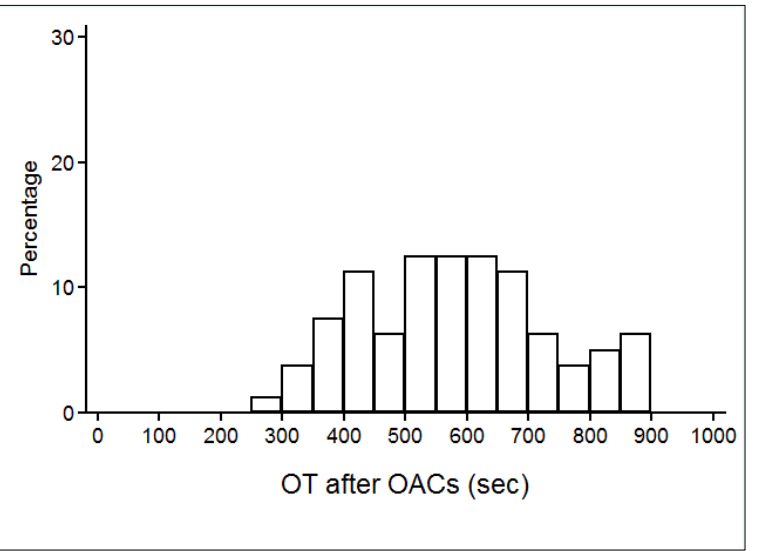

(D)

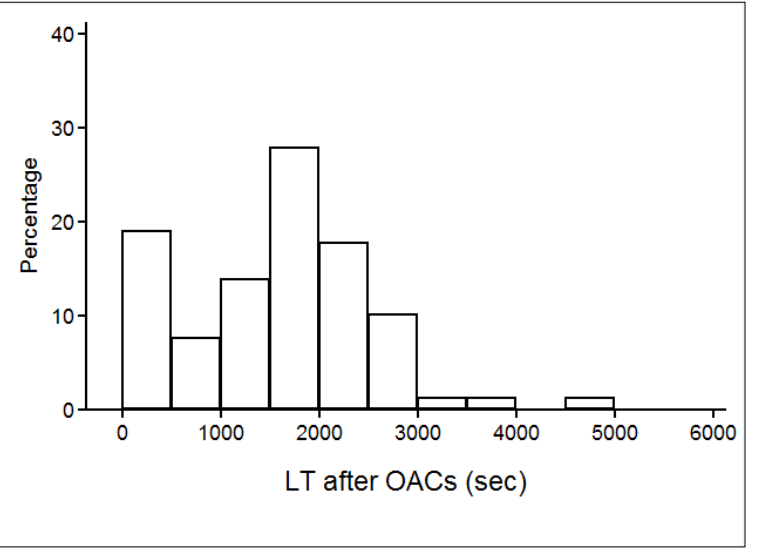


Figure 2: OT at baseline and after oral anticoagulants

The OT was significantly prolonged (less thrombotic) in patients who had been established on anticoagulation. The relative prolongation of OT from baseline was less with apixaban than with other NOACs or warfarin. Baseline OT is indicated by open bars; OT after anticoagulation is indicated by solid bars. $* p<0.01, \uparrow p<0.0001$.

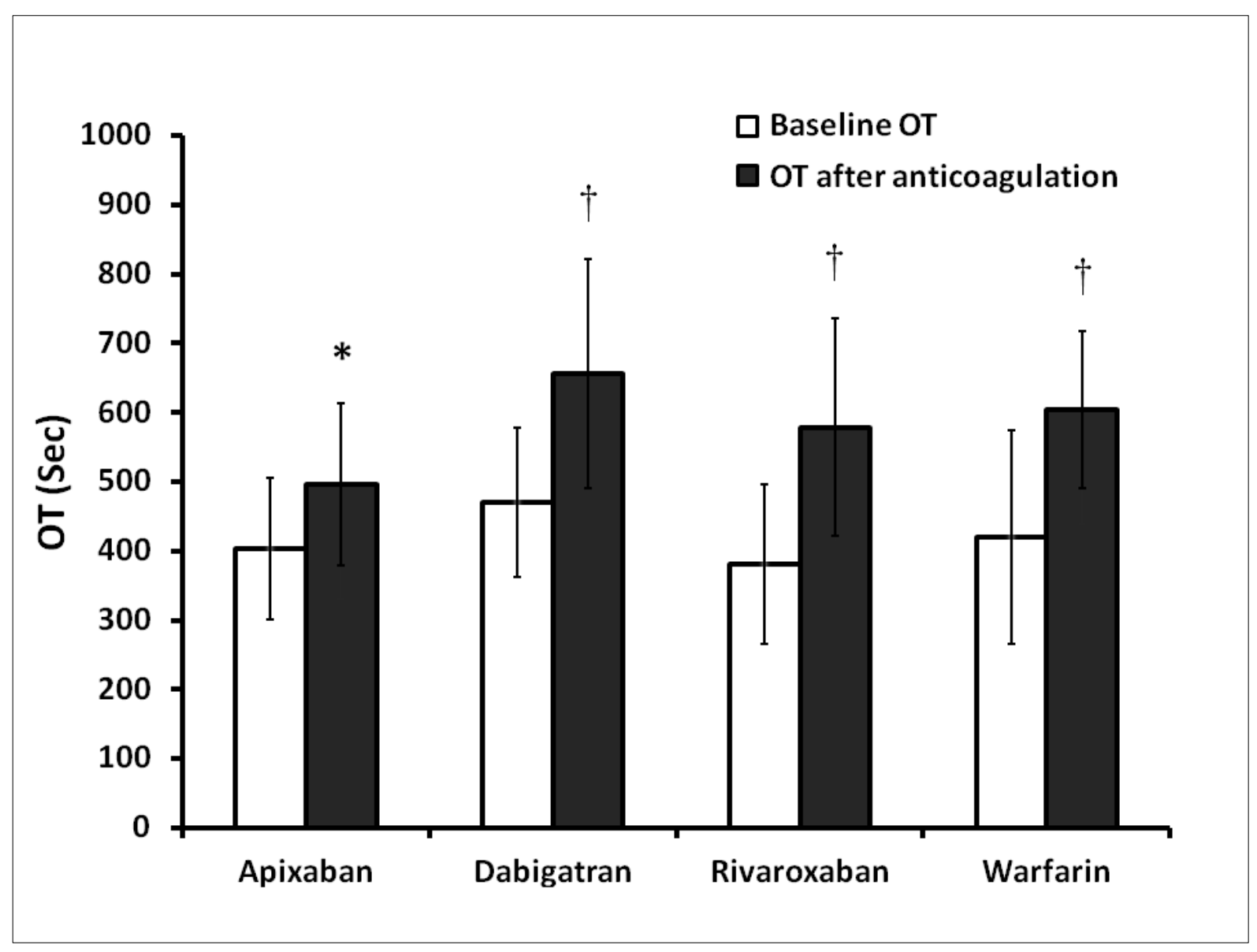


Figure 3: LT at baseline and after oral anticoagulants

Apixaban significantly reduced LT from baseline, but no significant difference in LT was observed with other NOACs or warfarin. Baseline LT is indicated by open bars; LT after anticoagulation is indicated by solid bars. ${ }^{*} p<0.01$.

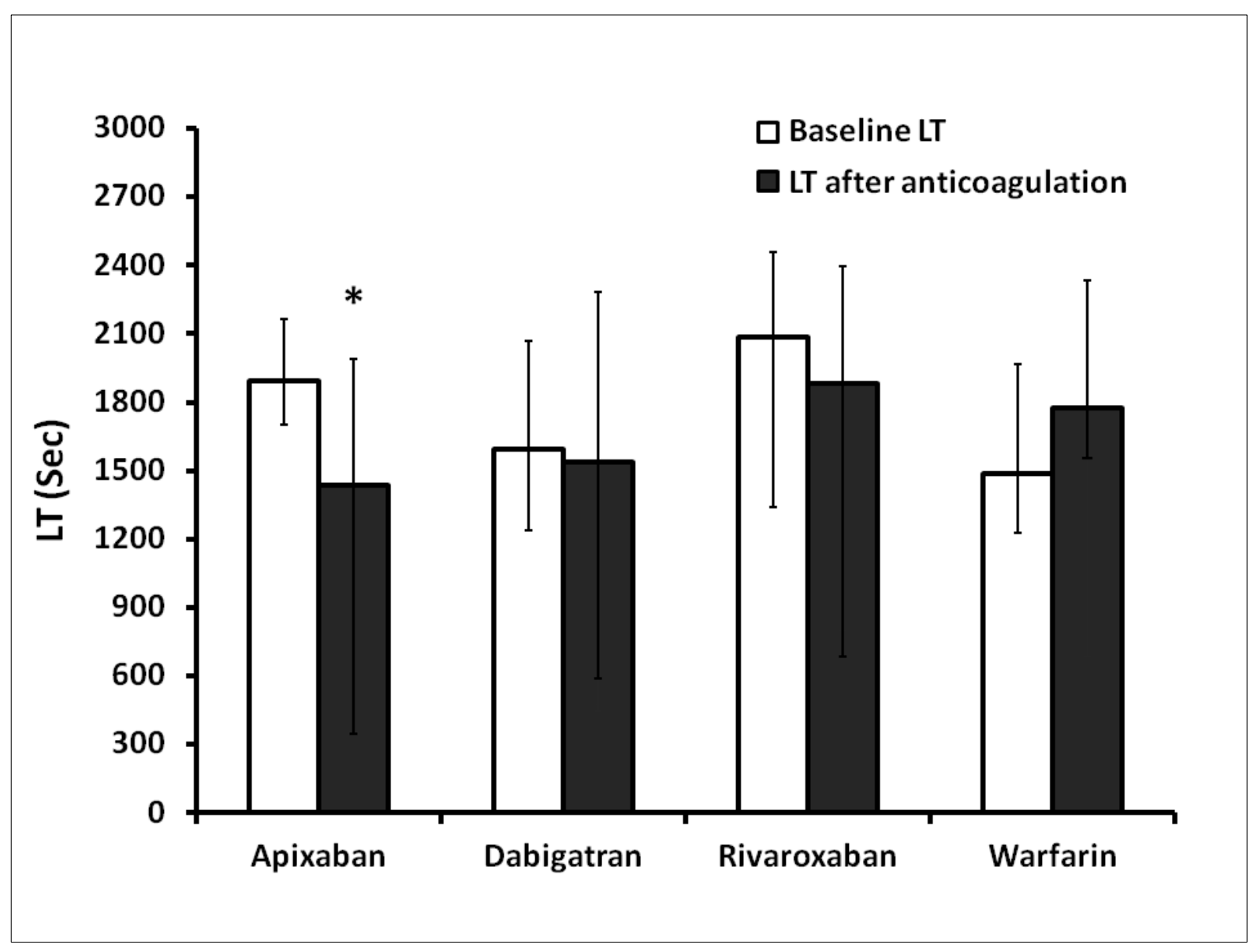

